\title{
Providing hierarchical approach for measuring supply chain performance using AHP and DEMATEL methodologies
}

\author{
Ali Najmi ${ }^{\mathrm{a}}$ and Ahmad Makui ${ }^{\mathrm{a}^{*}}$ \\ ${ }^{a}$ Department of Industrial Engineering, Iran University of Science and Technology, Tehran, Iran \\ A R T I C L E I N F O \\ Article history: \\ Received 15 April 2010 \\ Received in revised form \\ 15 May 2010 \\ Accepted 16 May 2010 \\ Available online 17 May 2010 \\ Keywords: \\ DEMATEL \\ Analytical Hierarchy Process \\ Supply Chain Performance \\ Integration \\ A B S T R A C T \\ Measuring the performance of a supply chain (SC) is normally of a function of various \\ parameters. Such a problem often involves in a multiple criteria decision making (MCDM) \\ problem where different criteria need to be defined and calculated, properly. During the past \\ two decades, Analytical hierarchy procedure (AHP) and DEMATEL have been some of the \\ most popular MCDM approaches for prioritizing various attributes. The study of this paper uses \\ a new methodology which is a combination of AHP and DEMATEL to rank various parameters \\ affecting the performance of the supply chain. The DEMATEL is used for understanding the \\ relationship between comparison metrics and AHP is used for the integration to provide a value \\ for the overall performance.
}

Metrics interdependence

\section{Introduction}

In the era of globalization, supply chains are being treated as extended enterprises. This arises from the attempts of the enterprises, being in different physical situations, and using the partners to gain competitive advantage. According to Jagdev and Browne (1998) supply chains are responsible for the entire lifetime of the product, from preparation of materials and supply management, to production and manufacturing, distribution and customer service, and ultimately recycling and disposal at the end of product life. In recent years, firms have realized the potential of supply chain management in the management of day to day operations. However, there are many firms without having enough insight for development of effective performance measures and metrics needed to achieve a fully integrated SCM. This is because they do not have the access to a balanced approach and a clear distribution between the metrics at strategic, tactical, and operational levels (Bhagwat \& Sharma, 2007). Measuring supply chain performance can facilitate a better understanding of the supply chain, positively influencing supply chain players' behavior, and improving its overall performance (Chen \& Paulraj, 2004). In order to achieve supply chain goal of fulfilling customer orders more quickly and efficiently than other competitors, a supply chain needs continuous improvements (Hausman, 2002). It demands that effective performance measurements be established and therefore, a system for measuring performance is required. Therefore, the purpose of calculating the performance, on one 
hand, is to give more information about the degree of achievement of the objectives and on the other hand, is to find actions to improve the values of metrics and consequently total performance (Berrah \& Cliville, 2007). In this sense, the so-called performance measurement systems are the necessary tools to support decision-making (Berrah \& Cliville, 2007). According to Berrah and Cliville (2007), a performance measurement system can be seen as a multi criteria tool, which is made by a set of metrics. A performance measurement system determines overall objectives and then, provides a series of performance metrics in order to achieve its objectives. If one can integrate metrics and other parameters, and achieve information about supply chain performance related to access organization goals, this model can be a support for decision-making. So the major problems in designing performance measurement systems include:

1. The identification of the performance structure, in which the parameters and necessary metrics for calculation the performance is identified.

2. The identification of the links between required parameters and metrics in relation to achieve the objectives.

The performance structure is widely considered in literature. In fact, most proposals provide criteria by which measure the performance. For example, Beamon (1998) categorizes performance measures in literature into two groups of qualitative and quantitative, so that customer satisfaction and responsiveness, flexibility, supplier performance, cost and other things for modeling supply chain are discussed. Beamon (1999) identified three types of performance measures as vital components for supply chain performance measurement system that includes resources, output, and flexibility. Gunasekaran et al. (2001) developed a framework for measuring the performance according to strategic, tactical and operational levels in supply chain. This framework deals with supplier, delivery, customer service, inventory and logistic costs. Hausman (2002) claimed that a supply chain needs to be evaluated by three criteria including service, asset and speed. Soni and Kodali (2010) for comparing performance of various supply chains and pointing out the poorly performing functions used the facilities, transportation, information, inventory, sourcing, and pricing categories of measurement which are proposed by Chopra and Meindl (2004). Felix et al. (2003) used a systematic process-based approach to build a model for measuring the overall performance of complex supply chains. Laura et al. (2007) developed a framework in which the reliability, responsiveness, flexibility, Re-configurability, and cost criteria have been proposed for measuring supply chain performance. Ashish Agarwal et al. (2006) employed a framework in which the market sensitiveness, process integration, information driver, and flexibility are used for measuring supply chain. The SCOR model (Supply Chain Council, 2006) was introduced in 1996 and includes 5 basic process including plan, source, make, deliver, and return. Kaplan and Norton (1992) proposed the balanced scorecard (BSC) approach as a method for performance evaluation through four perspectives of financial, internal business process, customer, and learning and growth. By using it can translate and convert the mission and organization strategies to the goals and measures in the above four perspectives. The problem of identification of the links between parameters is related to the integration of performance parameters. By literature review about performance score extraction in hierarchical models many works have been presented so far that can be classified to regular and irregular groups. Irregular integrated instruments are those that do not use a particular methodology, and authors using mathematical operators such as maximum, minimum, sum and so on, to integrate their model (Chang et al., 2007; Angerhofer \& Angelides, 2006; Berrah \& Cliville, 2007). Using the decision making and statistical methods to solve the models can be mentioned as examples of the regular integration instruments. Some of the researchers have used AHP methodology for integrating their models (Sharma et al., 2008; Bhagwat \& Sharma, 2007; Laura \& Roland, 2007; Sureshchandar \& Leisten, 2006). In this methodology, the interactions among the various parameters have not been considered. Sometimes ANP methodology was applied for considering interactions among the parameters of the model (Agarwal et al., 2006; Isik et al., 2007). The problem of using this methodology is too large number of matrices needed to extract the performance that actually make the work very complicated. 
In many cases, a combination of methodologies was used for integrating models (Taticchi et al., 2009; Yurdakul \& Ic, 2005; Banwet \& Deshmukh, 2008). By studying the methods used for the integration performance models, it is inferred that developing a methodology which is simple, and considers parameters interaction, and can be implemented practically, is required. Moreover, as seen before, most performance models or systems are unable to link performance metrics and organization strategy systematically. In addition, recognizing the metrics which are more important for accessing the target is not possible. Furthermore, critical metrics have high effects and need to more focus remains hidden from the managers' view. The metrics may also overlap which makes the performance score skewed. The primary focus of this paper is to propose a systematic approach to overcome the difficulties which are associated with measuring the performance of a supply chain. But before that, it is required to introduce the following two concepts related to performance measurement model:

Balance and its importance: Balance means considering different aspects of a case and avoiding unidimensional look at it. In other words, balance is, paying attention to all opinions based on their importance. For measuring supply chain performance, balancing is a key factor in its different levels which includes balance in the definition of strategy, balance in the chain performance measurement criteria, and the balance in the allocation metrics to performance criteria. For performance measurement and effective improvement, measurement goals must be balanced in accordance with the organizational strategy and show the organization objectives achievement level. Performance criteria must be balanced and evaluated under the organization's strategy and selected metrics need to reflect a balance between financial and non financial measures that could be related to strategic, tactical and operation levels.

Performance relativity: The performance and its criteria ought to be described as either good or bad. The performance is inherently asymptomatic and a comparative for performance determining must always exist. For example, consider that the prediction accuracy is an illustrative parameter of organization performance, it cannot be said that if 80 percent of predictions is executed properly, it is good or bad. It cannot even be said that if the percentage of prediction accuracy reach to 90 percent, there is really an improvement or not. For the interpretation of these data, organizations must know (or guess) that what the competitors have done in a similar process, or how much the improvement of a process in the organization is in comparison with that of competitor organization. Therefore, the concept of performance will find meaning only in comparison. In other words, performance is a relative concept and needs judgment and interpretation. According to this description, comparing of a supply chain with a competitor supply chain or a virtual supply chain as an ideal pattern to evaluate it is essential.

\section{Proposed framework for creating performance structure}

Proposed framework is used for evaluation of supply chain via a series of chain characteristics and based on organization strategies, with another supply chain with the same strategy, by use of metrics. Therefore, the most important model pillar is extraction of organization strategy and forming the model based on it. Based on organization strategy, appropriate metrics are selected for a chain and necessary arrangements are provided to compare the supply chain with ideal supply chain. This framework has four levels: 1) The strategic level in which the organization strategy will be determined, 2) Supply chain performance criteria level in which the importance of each chain performance criteria for accessing the strategic objectives is defined, 3) The metrics level that include some performance indicators for performance calculation and 4) Chain level in which the benchmark chain based on performance attributes is compared to ideal chain and will be evaluated. 
Strategic level: Strategy factors and consequently the supply chain strategy lie at this level. The strategic goals, express the balanced needs of organization stakeholders. The most important stakeholders of an organization are: shareholders, customers, employees, business partners (contractors, suppliers) and the society. If there is incompatibility between the organization and supply chain strategies, it is unlikely to optimized supply system. The strategy of each organization is different from other organization based on its status and conditions. For this level can used axes, provided by the strategic approaches such as BSC.

Supply chain performance criteria level: Supply chains performance is measured by criteria which are particular for the supply chain and achieving them will help the chains to achieve goals. In other words, the measures which a supply chain must encompass to achieve strategic objectives, are placed at this level. For this level, one can use axes provided by the models such as SCOR which are related to supply chains and offers evaluation criteria for SC performance. In fact, criteria in this level express the balanced needs of supply chain's stakeholders. These stakeholders include suppliers, manufacturers, distributors, and retailers in different levels.

Metrics level: In this level, the desired metrics are situated to evaluate the performance. These metrics are selected based on existing groups in the performance criteria level and their selection is different for various chains and organizations. Note that the metrics always must be selected properly, and cover the three levels of strategic, tactical and operational. We need to pay attention that metrics are selected as balanced, and they cover the three-level of strategic, tactical and operational. According to Rushton and Oxley (1989), such a hierarchy is based on the time horizon for activities and the pertinence of decisions and influence of different levels of management. Strategic measures affect the top level management decisions, often reflecting extensive studies of policies, programs, corporate financial plans, competition and conform to the organizational goals. The tactical level deals with resource allocation and performance measurement of goals which could be achieved regarding access of results specified at the strategic level. Measuring performance in this level provides valuable feedback for mid-level management decisions. Metrics and measurements of operational level require accurate data and evaluation of low level managers' decisions results. Superintendents and staffs are moving towards achievement of operational objectives, which, if fulfilled, will lead to achievement of tactical objectives (Gunasekaran et al., 2004).

Enough attention must be paid on the number of metrics which are selected for performance extraction. Large number of metrics is not a reason for accuracy of final performance. Most companies have a large number of performance measures which have emerged based on the suggestions from staff and consultants. They fail to realize that performance assessment will be better by using a trivial few (in fact not quite partial, but those that are critical for success) (Gunasekaran et al., 2004).

Chains level: In the proposed framework, two supply chain are compared and based on that, the performance of the supply chain (under study) is calculated with respect to another one (ideal). Performance comparison prerequisite of two organizations or two supply chains is similarity of their classes or strategies. Thus, the supply chains which sit in the chain level must have similar strategies. Ideal supply chain can be a virtual chain which is determined based on the benchmark supply chain objectives and make possible the access to them. In other words, benchmark supply chain is compared with the ideal supply chain. This framework is shown in figure 1 . In this figure, A is the total number of determinants, $\mathrm{J}$ is the total number of criteria, and $\mathrm{M}_{\mathrm{jk}}$ is the $k$-th metric under criterion j.

\subsection{Mutual dependence of metrics}

One of the objectives of this study is to provide a method to calculate the performance of a supply chain with respect to another supply chain as an ideal pattern, with regard to the interaction of 
comparable criteria. Metrics of the framework are those which help accessing to the controlling criteria of supply chain performance. Therefore, these are dependent on the criteria. However, there is some mutual dependence among the metrics. Dotted line shown in figure 1 depicts this issue. Values of this dependency are extracted by the use of DEMATEL method and experts’ opinions.

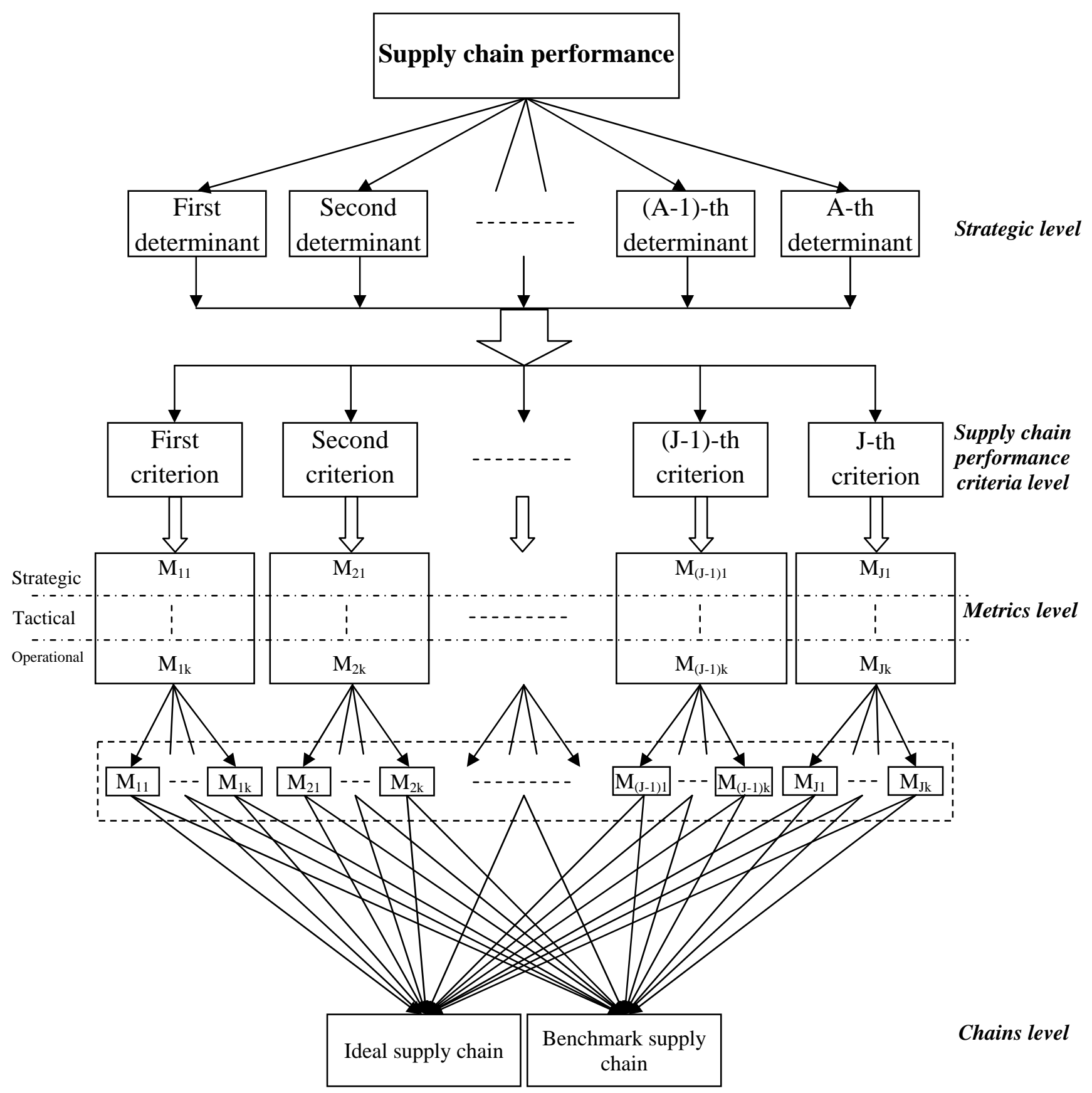

Fig. 1. Proposed framework for extraction of performance structure

\section{Combined methodology for identification of the links}

For comparing between the benchmark chain and ideal chain and providing a numeric comparative performance, the composition of hierarchical analysis process (AHP) method and DEMATEL method is used. AHP provides the possibility that a set of complicated issues, are compared by the relative importance of each issues relative to its impact on the solution of the problem. The AHP 
(Saaty, 1980) is a tool used for solving multi-criteria decision-making problems and develops a framework for coping with the palpable and non palpable, quantitative and qualitative aspects. It consists of three main steps:

1. Decomposing the problem into a hierarchy of different levels of elements

2. Application of a measurement methodology for extracting the elements priority

3. Combination of elements priority to extract the final decision

The AHP helps to rank and decide in a systematic and rational way. AHP also enables the analyzer to evaluate the goodness of judgment with the consistency ratio (CR). A CR of 0.10 or less indicates a consistent and acceptable judgment (Kim, 2006). Although AHP is a powerful and flexible decisionmaking technique that helps decision-makers to set priorities and select the best alternative, but the remarkable weakness of AHP is that it cannot deal with interconnections among the decision factors at same levels, because the decision framework in the AHP assumes a one-way hierarchical relationship between decision levels. In many issues that interactions among the decision variables exist, AHP is not effective to use (Isik et al., 2007). With combining this method and DEMATEL method we can solve the above problem. DEMATEL Method (Fontela \& Gabus, 1974) created by the Battelle Geneva Association is based on the concept of pair-wise comparison of decision characteristics such as solutions alternative, criteria, and etc. These attributes are compared by the relative influence. In this article discrete assessment scale 0-4 is applied. Zero means no influence and 4 indicates extreme influence of the first attribute compared to the other one. Scales between these two numbers indicate intermediate states between these two influences. Numerical representation of direct influences can be displayed as matrix $A$. Number of rows and columns of this matrix is equal to number of compared attributes $(n)$. The $i$-th row of $A$ shows direct influence of $i$-th attribute on all attributes including itself. This influence can indicate the relative importance of attributes. Furthermore, the $j$-th column indicates the influence of all attributes on $j$-th attribute. Matrix $A$ can be normalized by the following formula:

$$
\begin{aligned}
& N=\frac{A}{\max _{i} \sum_{j=1}^{n} a_{i j}}, \quad(i, j=1,2, \ldots, n) \\
& T=N+N^{2}+\ldots=N(I-N)^{-1},
\end{aligned}
$$

where operator max denotes the maximum row-wise sum of matrix $A$ and $I$ denotes the identity of matrix $\mathrm{n} \times \mathrm{n}$. Matrix $T$ shows direct and indirect relations of elements. Therefore, by calculating matrix $T$, we can extract the mutual dependence between the metrics. The reasons of using this combination approach for solving the proposed structure are as follows: 1) Many factors, metrics and criteria in decision environment are interdependent from one another, 2) Some criteria and metrics are subjective and measuring them are difficult, and 3) This composition provides a numerical measure for performance.

\section{Data gathering using expert opinion}

The relative weights in the pair wise comparison matrices of AHP, is achieved through discussion with experts group of benchmark supply chain. The group includes experts which have extensive experience in supply chain management. To obtain the relative weights, a scale of 1-9 provided by Saaty (1980) is used where 1 indicates equal impact and 9 indicates the highest impact of row element than column element. If the experts feel that element of the column has higher impact than the row element, thus reverse the numbers from 1 to 9 is assigned. In order to obtain the relative 
weights of strategic determinants, several questions can be asked by the researcher. A sample question is as following: "What is the relative impact on the organization strategy and consequently on the supply chain performance, when determinant $A$ is compared to the determinant $B$ ". Moreover, to achieve the relative weights of supply chain performance criteria, the following question can be posed by the researcher: "What is the relative impact of criterion $A_{1}$ on determinant $A$ when compared with criterion $A_{2}$ ". Questions that can be posed to achieve relative weights measures of the metrics level, under specific criterion of supply chain level with the aim of achieving specified determinant at strategic level are as follows: "With the aim of maximizing determinant $A$, what is the relative impact on the criterion $A_{1}$ by metric $A_{11}$, when compared to metric $A_{12}$ ". Also, the weights related to chain position from point of view of the extracted metrics can be collected as follows: "With the goal of maximizing determinant $A$, from point of view of metric $A_{11}$, what is the relative impact of ideal chain with respect to the benchmark chain?". And finally, to extract matrix $A$ in order to extraction of interactions, the following question can be posed by the researcher: "What is the effect of metric $A_{11}$ on the metric $A_{12}$ with the aim of maximizing determinant $A$ ?". A graphical summary of the combined model and its decision environment related to performance of the supply chain is shown in figure 1. The aim of using AHP and DEMATEL is providing a performance score by comparison between the two supply chains to help supply chain management decision-making.

\section{The application of combined methodology for integration}

The steps required to solve the proposed framework by using AHP and DEMATEL methodologies are as follows:

\section{Step 1: Placement of framework elements and problem structuring}

The top elements of framework hierarchy are decomposed into sub criteria and metrics. For the development of the framework, it is necessary to identify the parameters at each level (determinants, criteria and metrics) and provide a definition of them and communications between them. The ultimate objective of this hierarchy is to provide a score for a supply chain performance of an organization with respect to another one. In order to do this, the same numbers of hierarchies as those of elements extracted at the strategic level are studied and then a weighted index for the performance of supply chain is calculated.

\section{Step 2: Pair-wise comparison matrices between parameters at different levels}

On a scale of 1 to 9, a series of questions are asked by the decision maker to answer pair-wise comparison with respect to a higher level. These questions are used for extraction of the relative weights of parameters used in the framework by AHP.

\section{Step 3: Matrices of relative influences for extraction of mutual dependences}

To reflect the mutual dependence between the metrics, information of the influences of metrics on each other will be collected. For performing these comparisons, the scale of 0 to 4 is used. In this scale, zero implies no influence, whereas 4 show the extreme influence of row element with respect to column element. Number of necessary matrices depends on the number of determiant used in the strategic level. One matrix for each determinant is required.

\section{Step 4: Using DEMATEL for calculation of interdependencies}

Matrix of direct and indirect relationship $T$, is obtained through the formulas (1) and (2). By using these formulas the effects of a metric on the other metrics can be calculated. 
Desirability index equation for chain $i$ and determinant $a$ is defined as formula (3):

$D I_{i a}=\sum_{j=1}^{J} \sum_{k=1}^{K j a} D_{j a} M_{k j a}^{D} M_{k j a}^{I} S_{i k j a}$,

where $D_{j a}$ is the relative importance weight of criteria $j$ under the determinant $a, M_{k j a}^{D}$ is the relative importance weight of metric $k$ under the criterion $j$ with respect to determinant $a, M_{k j a}^{I}$ is the relative weight of metric $k$ of the criterion $j$ and under determinant $a$ for interdependencies of metrics, $S_{i k j a}$ is the relative impact of chain $i$ under the metric $k$ criterion $j$ and the network hierarchy $a, K_{j a}$ is the total number of metrics for criterion $j$ and determinant $a$, $\mathrm{J}$ is the total number of criteria. Table 1 shows the required calculations for the desirability indices of chains under a determinant and the results are obtained by using the weights from the pair-wise comparisons of the chains, criteria, metrics, and weights results from interdependencies of metrics by DEMATEL method.

The second column in Table 1 is obtained from the results of step 2, which is based on relative impact of criteria under the selected determinant from strategic level. The weights which are obtained from pair-wise comparison matrix for the relative impact of metrics under supply chain criteria are displayed in Column 4. The fifth column values, shows the interdependent weights related to metrics. Relative weights of two chains for each metric are given in the sixth and seventh columns of Table 1. These weights are achieved by comparing two chains for each chain performance metric. The last two columns show the desirability index of each supply chain for each metric. For each of supply chains under determinant a, the summation of this results is calculated in the final row of Table 1 . By use of these two values the performance score of benchmark chain can be calculated with respect to the ideal chain based on the selected determinant.

\section{Table 1}

Supply chain performance desirability index for a selected determinant from strategic level

\begin{tabular}{|c|c|c|c|c|c|c|c|c|}
\hline •. & $\begin{array}{c}\text { Criterion } \\
\text { weight }\end{array}$ & Metric & $\begin{array}{c}\text { Relative } \\
\text { importance } \\
\text { weight for } \\
\text { metrics }\end{array}$ & $\begin{array}{l}\text { Weight of } \\
\text { Mutual } \\
\text { dependence } \\
\text { of metrics }\end{array}$ & $\begin{array}{l}\text { Weights of } \\
\text { ideal chain } \\
\text { for each } \\
\text { metric }\end{array}$ & $\begin{array}{l}\text { Weights of } \\
\text { benchmark } \\
\text { chain for } \\
\text { each metric }\end{array}$ & Ideal chain & Benchmark chain \\
\hline 1 & $\begin{array}{c}D_{1 a} \\
\vdots \\
D_{1 a}\end{array}$ & $\begin{array}{c}1 \\
\vdots \\
k_{1 a}\end{array}$ & $\begin{array}{c}M_{11 a}^{D} \\
\vdots \\
M_{k_{1 a}}^{D}\end{array}$ & $\begin{array}{c}M_{11 a}^{I} \\
\vdots \\
M_{k_{1 a}}^{I}\end{array}$ & $\begin{array}{c}S_{111 a} \\
\vdots \\
S_{1 k_{1 a}}\end{array}$ & $\begin{array}{c}S_{211 a} \\
\vdots \\
S_{2 k_{1 a}}\end{array}$ & $\begin{array}{c}D_{1 a} M_{11 a}^{D} M_{11 a}^{I} S_{111 a} \\
\vdots \\
D_{1 a} M_{k_{1 a}}^{D} M_{k_{1 a}}^{I} S_{1 K_{1 a}}\end{array}$ & $\begin{array}{c}D_{1 a} M_{11 a}^{D} M_{11 a}^{I} S_{211 a} \\
\vdots \\
D_{1 a} M_{k_{1 a}}^{D} M_{k_{1 a}}^{I} S_{2 K_{1 a}}\end{array}$ \\
\hline 2 & $\begin{array}{c}D_{2 a} \\
\vdots \\
D_{2 a}\end{array}$ & $\begin{array}{c}1 \\
\vdots \\
k_{2 a}\end{array}$ & $\begin{array}{c}M_{12 a}^{D} \\
\vdots \\
M_{k_{2 a}}^{D}\end{array}$ & $\begin{array}{c}M_{12 a}^{I} \\
\vdots \\
M_{k_{2 a}}^{I}\end{array}$ & $\begin{array}{c}S_{112 a} \\
\vdots \\
S_{1 k_{2 a}}\end{array}$ & $\begin{array}{c}S_{212 a} \\
\vdots \\
S_{2 k_{2 a} a}\end{array}$ & $\begin{array}{c}D_{2 a} M_{12 a}^{D} M_{12 a}^{I} S_{112 a} \\
\vdots \\
D_{2 a} M_{k_{2 a}}^{D} M_{k_{2 a}}^{I} S_{1 K_{2 a}}\end{array}$ & $\begin{array}{c}D_{2 a} M_{12 a}^{D} M_{12 a}^{I} S_{212 a} \\
\vdots \\
D_{2 a} M_{k_{2 a}}^{D} M_{k_{2 a}}^{I} S_{2 K_{2 a}}\end{array}$ \\
\hline$\vdots$ & $\vdots$ & $\vdots$ & $\vdots$ & $\vdots$ & $\vdots$ & $\vdots$ & $\vdots$ & $\vdots$ \\
\hline$J$ & $\begin{array}{c}D_{J a} \\
\vdots \\
D_{J a} \\
\end{array}$ & $\begin{array}{c}1 \\
\vdots \\
k_{J a} \\
\end{array}$ & $\begin{array}{c}M_{1 J a}^{D} \\
\vdots \\
M_{k_{J a}}^{D} \\
\end{array}$ & $\begin{array}{c}M_{1 J a}^{I} \\
\vdots \\
M_{k_{J a}}^{I} \\
\end{array}$ & $\begin{array}{c}S_{11 J a} \\
\vdots \\
S_{1 k_{J a}} \\
\end{array}$ & $\begin{array}{c}S_{21 J a} \\
\vdots \\
S_{2 k_{J a}} \\
\end{array}$ & $\begin{array}{c}D_{J a} M_{1 J a}^{D} M_{1 J a}^{I} S_{11 J a} \\
\vdots \\
D_{J a} M_{k_{J a}}^{D} M_{k_{J a}}^{I} S_{1 K_{J a}} \\
\end{array}$ & $\begin{array}{c}D_{J a} M_{1 J a}^{D} M_{1 J a}^{I} S_{21 J a} \\
\vdots \\
D_{J a} M_{k_{J a}}^{D} M_{k_{J a}}^{I} S_{2 K_{J a}}\end{array}$ \\
\hline Sun & & & & & & & $\sum_{j=1}^{J} \sum_{k=1}^{K j a} D_{j a} M_{k j a}^{D} M_{k j a}^{I} S_{1 k j a}$ & $D I_{2 a}=\sum_{j=1}^{J} \sum_{k=1}^{K j a} D_{j a} M_{k j a}^{D} M_{k j a}^{I} S_{2 k j a}$ \\
\hline
\end{tabular}

\section{Step 6: Calculation of supply chain performance weighted index (PWI)}

To complete analysis, supply chain performance weighted index is calculated for each chain. The supply chain performance weighted index for a chain, is equal to the multiplication of the desirability indices by the relative importance weights of the determinants. The relative importance weight of 
determinants is obtained from Step 2. The calculation of this step is shown in Table 2. Where $D_{a}$ is the relative importance weight of determinant $a$ on the organization strategy and consequently on the supply chain performance.

Table 2

Supply chain performance weighted index (PWI) for chains

\begin{tabular}{cccccc}
\hline Determinants & $\begin{array}{c}\text { Weights of } \\
\text { determinants }\end{array}$ & $\begin{array}{c}\text { Desirability indices } \\
\text { for benchmark SC }\end{array}$ & $\begin{array}{c}\text { Desirability indices } \\
\text { for ideal SC }\end{array}$ & $\begin{array}{c}\text { Weighted Index } \\
\text { for benchmark SC }\end{array}$ & $\begin{array}{c}\text { Weighted index } \\
\text { for ideal SC }\end{array}$ \\
\hline 1 & $D_{1}$ & $D I_{21}$ & $D I_{11}$ & $D_{1} * D I_{21}$ & $D_{1} * D I_{11}$ \\
2 & $D_{2}$ & $D I_{22}$ & $D I_{12}$ & $D_{2} * D I_{22}$ & $D_{2} * D I_{12}$ \\
& $\vdots$ & $\vdots$ & $\vdots$ & $\vdots$ & $\vdots$ \\
$\vdots$ & $D_{a}$ & $D I_{2 a}$ & $D I_{1 a}$ & $D_{a} * D I_{2 a}$ & $D_{a} * D I_{1 a}$ \\
& $\vdots$ & $\vdots$ & $\vdots$ & $\vdots$ \\
A & $D I_{2 A}$ & $D I_{1 A}$ & $D_{A} * D I_{2 A}$ & $D_{A} * D I_{1 A}$ \\
\hline
\end{tabular}

Step 7: Extracting importance of metrics

To complete the analysis and to obtain the critical metrics, the importance index of metrics for determinant $a$ and metric $k$ for each metric is defined as follows:

$C_{k a}=D_{j a} M_{k j a}^{D} M_{k j a}^{I} *\left[1-S_{2}\right]$

Where $\mathrm{C}_{\mathrm{ka}}$ is the importance of metric $k$ under determinant $a$, and $S_{2}$ is weight of the benchmark chain under metric $k$. The first part of the above formula shows the importance of metric $k$, and the second part shows the distance between benchmark chain and the ideal chain. According to the above formula, the more important the metric or the distance between benchmark chain and the ideal chain is, the more important the desired metric is.

Step 8) Calculation of the importance index of metrics (IIM)

In the previous step, the importance of the metrics under one determinant is identified. It is necessary to obtain a score for overall importance so that the effect on all the determinants is calculated. Therefore, the importance index of metrics for each metric is calculated. Importance index for a metric is the product of the importance indices and the relative importance weights of determinants of the supply chain performance. Table 3 shows the calculation of this step. 
Table 3

The importance index of metrics (IIM)

\begin{tabular}{cccc}
\hline Determinant & Weights of determinants & Importance of metrics & Importance index of metrics \\
\hline 1 & $D_{1}$ & $C_{1}$ & $D_{1} * C_{1}$ \\
2 & $D_{2}$ & $C_{2}$ & $D_{2} * C_{2}$ \\
& $\vdots$ & $\vdots$ & $\vdots$ \\
& $D_{a}$ & $C_{a}$ & $D_{a} * C_{a}$ \\
& $\vdots$ & $\vdots$ & $\vdots$ \\
S & $D_{A}$ & $C_{A}$ & $D_{A} * C_{A}$ \\
\hline
\end{tabular}

The metrics which have high importance index are more important for chain management and to improve the performance score, managers must put their priority on their improvement. The schematic of presented approach is shown in figure 2.

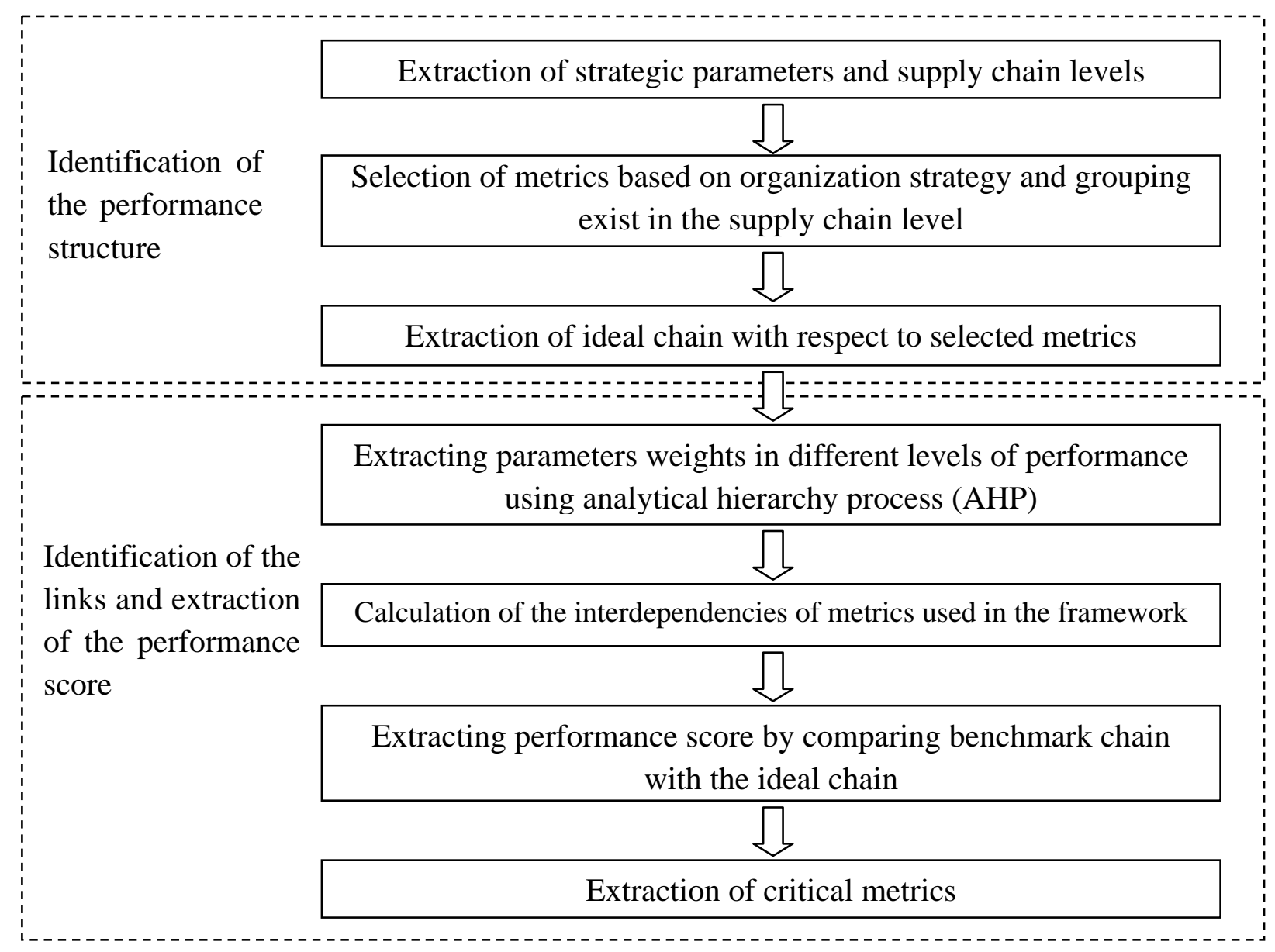

Fig. 2.Schematic of extracting performance score for a supply chain

\section{Advantages of the proposed method}

Advantages of the proposed framework can be stated as follows:

- It can be aligned with organization strategy easily. 
- It is balanced from any direction (Strategic determinant, chain performance criteria, and metrics level).

- It can be integrated easily and provide a score for chain performance.

- It is possible to avoid the increase of the metrics focusing on the critical metrics.

- It is an extractive performance index that can study the sensitiveness of the activities.

- It is also an approach to extract the importance of attribute and metrics for accessing business goals and strategy is defined.

- $\quad$ The mutual dependence between the metrics is included in the framework and is tried to keep extracted performance score closer to reality.

\section{Case Study}

Proposed framework for an automotive company in Tehran with production capacity of three hundred thousand years is investigated. In discussion with relevant experts, parameters of financial, customer, internal business processes, environmental, and learning and growth were extracted as fundamental determinants in the strategic level. Also the parameters of flexibility, reliability, responsiveness, quality, and asset management that are supply chains' characteristics, and satisfying them make it possible to achieve strategic determinants, was chosen for the supply chain level. In addition, in order to measure the achievement of each selected criterion, the metrics given in Table 4 were used.

\section{Table 4}

Selected metrics to measure the achievement of each criterion

\begin{tabular}{ll}
\hline Criteria & Metrics \\
\hline \multirow{3}{*}{ Flexibility } & Downside SC adaptability \\
& Raw materials inventory level \\
& Operational flexibility \\
\hline \multirow{3}{*}{ Reliability } & Perfect order fulfillment \\
& Total order value according to orders \\
& Waiting times \\
\hline \multirow{2}{*}{ Responsiveness } & Lead time \\
& On-time delivery \\
& Produce and test cycle time \\
\hline \multirow{2}{*}{ Quality } & Customer service level \\
& Communication \\
\hline
\end{tabular}

After solving the framework, it was observed that the customer determinant has the most impact (weight 0.544 ) on the performance score. The performance score obtained from comparing this chain and ideal chain showed that benchmark chain performance with compare to ideal chain is 23.7 percent. In addition, calculation of importance index of metrics, as shown in figure 3, indicates that 
the importance of communication metric is the most and equal to 0.0125 . Therefore, management should focus more on improving communication in the supply chain. Another important metric is customer service level. In addition to the above metrics, the metrics which are of second importance level include cash flow, perfect order fulfillment, waiting times, lead time and on-time delivery.

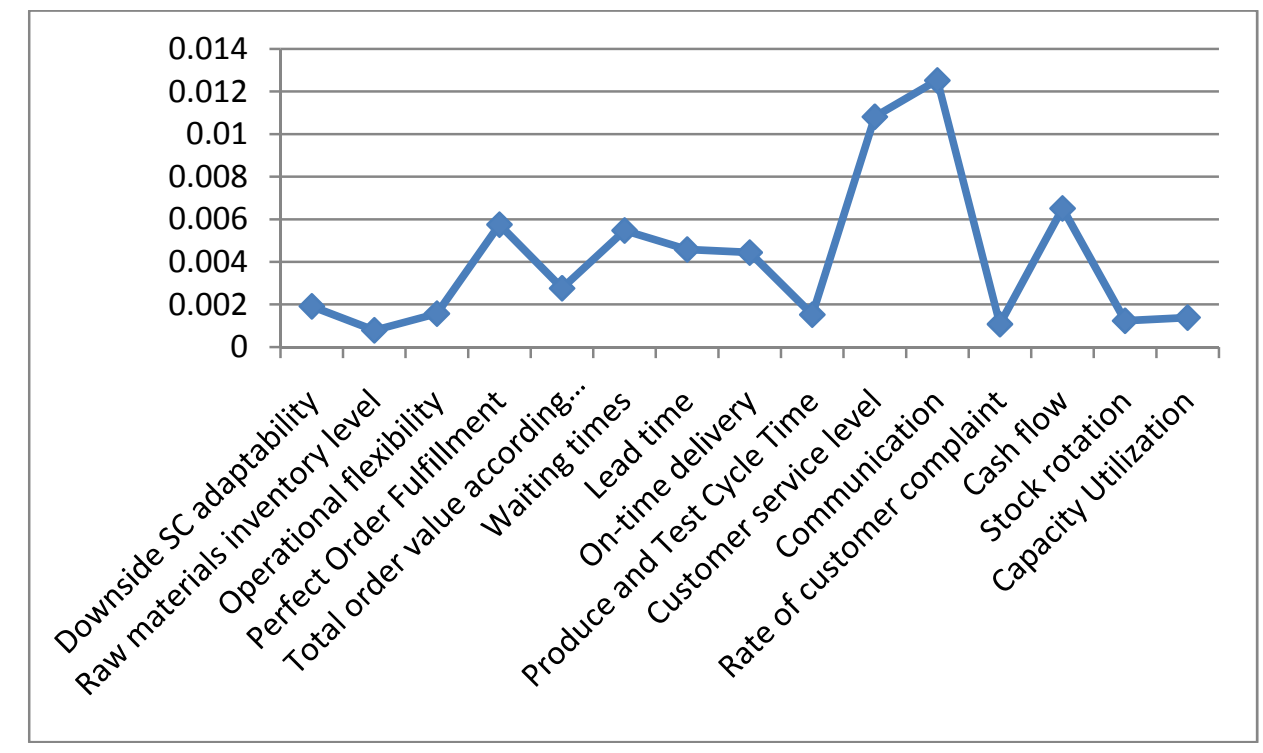

Fig. 3. Importance index of metrics

\section{Conclusion}

This paper aims to help managers in supply chain for decision making. First, the key concepts and methods of supply chain performance measurement and work done in this field were reviewed. It was observed that although the large number of researchers emphasized on the importance of measuring and has provided approaches and methods, there is lack of a comprehensive framework that could link the strategy to the performance measures considering supply chain characteristics. Moreover, creating a methodology for integration and extraction of a score for performance with regard to mutual dependence of parameters was seemed to be of great importance and could be considered. In proposed framework, the position of required factors for measuring performance is indicated, and with regard to organization strategy, the present benchmark chain is compared with ideal chain of the same class. In order to extract the performance and identify the most important factor, the combined methodology of the AHP and DEMATEL are used.

\section{References}

Agarwal, A., Shankar, R. \& Tiwari, M.K. (2006). Modeling the metrics of lean, agile and leagile supply chain: An ANP-based approach, European Journal of Operational Research, 173, 211225.

Angerhofer, B. J. \& Angelides, M. C. (2006). A model and a performance measurement system for collaborative supply chains, Decision Support Systems, 42, 283- 301.

Banwet, D.K. \& Deshmukh, S.G. (2008). Evaluating performance of national R\&D organizations using integrated DEA-AHP technique, International Journal of Productivity and Performance Management, 57(5), 370-388.

Beamon, B. M. (1999). Measuring Supply Chain Performance, International Journal of Operations and Production Management, 19(3), 275-292.

Beamon, B. M. (1998). Supply chain design and analysis: models and methods, International Journal of Production Economicst, 55(3), 281-294. 
Berrah, L. \& Cliville', V. (2007). Towards an aggregation performance measurement system model in a supply chain context. Production Planning \& Control, 58, 709-719.

Bhagwat, R. \& Sharma, M.K. (2007). Performance measurement of supply chain management using the analytical hierarchy process, Computers in Industry, 18(8), 666-680.

Chen, I.J. \& Paulraj, A. (2004). Understanding supply chain management: critical research and a theoretical framework. International Journal of Production Research, 42(1), 131-163.

Chang, S.L., Wang, R.C. \& Wang, S.Y. (2007). Applying a direct multi-granularity linguistic and strategy-oriented aggregation approach on the assessment of supply performance. European Journal of Operational Research, 177, 1013-1025.

Chopra, S. \& Meindl, P. (2004), Supply Chain Management: Strategy, Planning and Operations, Prentice-Hall, Upper Saddle River, NJ.

Felix T.S. Chan \& Qi, H. J. (2003), An innovative performance measurement method for supply chain management, Supply Chain Management, 8(3), 209-223.

Fontela, E. \& Gabus, A. (1974), DEMATEL. Progress Achieved, Futures 6, 329-333.

Gunasekaran, A., Patel, C. \& Tirtiroglu, E. (2001). Performance measures and metrics in a supply chain environment, International Journal of Operations \& Production Management, 21(1) 71-87.

Gunasekaran, A., Patel, C., Ronald, E., \& McGaughey, R. (2004). A framework for supply chain performance measurement, International Journal of Production Economics, 87(3), 333-348.

Hausman, Warren H. (2002), Supply chain performance metrics, Corey Billington, Terry H., Hau L., and Hohn N. (Ed.), The practice of supply chain management, Kluwer Academic Publishiers.

Isik, Z., Dikmen, I. \& Birgonul, M. T. (2007). Using Analytic Network Process (ANP) for Performance Measurement in Construction, The construction and building research conference of the Royal Institution of Chartered Surveyors, Georgia Tech, Atlanta USA.

Jagdev, H.S. \& Browne, J. (1998), The extended enterprise-a context for manufacturing, Production Planning \& Control, 9(3), 216-229.

Kaplan, R.S. \& Norton D.P. (1992). The Balanced Scorecard - Measures that drive performance, Harvard Business Review, 71-79.

Kim, Y. H. (2006 ). Study on Impact Mechanism for Beef Cattle Farming and Importance of Evaluating Agricultural Information in Korea Using DEMATEL, PCA and AHP. Agricultural Information Research, 15(3), 267-280.

Laura XU Xiao Xia, Bin MA, Roland LIM, (2007). AHP Based Supply Chain Performance Measurement System, Singapore Institute of Manufacturing Technology, Singapore.

Rushton, A. \& Oxley, J. (1989). Handbook of Logistics and Distribution Management. Kogan Page Ltd., London.

Saaty, T. L. (1980). The Analytic Hierarchy Process. McGraw- Hill, New York.

Sharma, M. J., Moon, I. \& Bae, H. (2008). Analytic hierarchy process to assess and optimize distribution network, Applied Mathematics and Computation, 202, 256-265.

Soni, G. \& Kodali, R. (2010). Internal benchmarking for assessment of supply chain performance, Benchmarking: An International Journal, 17(1), 44-76.

Supply Chain Council (2006). Supply Chain Operations Reference Model: Overview of SCOR Version 8.0, available at: www.Supply Chain.org

Sureshchandar, G.S. \& Leisten, R. (2006). A framework for evaluating the criticality of software metrics: an analytic hierarchy process (AHP) approach, Measuring Bussiness Execellence, 10(4) 22-33.

Taticchi, P., Tonelli, F. \& Cagnazzo, L. (2009). A decomposition and hierarchical approach for business performance measurement and management, Measuring Bussiness Execellence, 13(4), 47-57.

Yurdakul, M. \& Ic, Y. T. (2005), Development of a performance measurement model for manufacturing companies using the AHP and TOPSIS approaches, International Journal of Production Research, 43(21), 4609-4641. 